International Journal of Pure and Applied Mathematics

Volume 112 No. $4 \quad 2017,695-708$

ISSN: 1311-8080 (printed version); ISSN: 1314-3395 (on-line version)

url: http://www.ijpam.eu

doi: 10.12732 /ijpam.v112i4.4

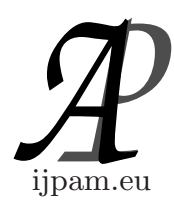

\title{
DYNAMICS OF AN SIS EPIDEMIC MODEL WITH \\ A SATURATED INCIDENCE RATE UNDER TIME DELAY AND STOCHASTIC INFLUENCE
}

\author{
Ranjith Kumar G. ${ }^{1}$, Lakshmi Narayan K. ${ }^{2}$, Ravindra Reddy B. ${ }^{3}$ \\ ${ }^{1}$ Department of Mathematics \\ Anurag Group of Institutions \\ Hyderabad, INDIA \\ ${ }^{2}$ Department of Mathematics \\ Vignan Institute of Engg. \& Tech. \\ Hyderabad, INDIA \\ ${ }^{3}$ Department of Mathematics \\ JNTU College of Engg. \\ Jagityal, Karimnagar, INDIA
}

\begin{abstract}
This paper examines an SIS model with saturated incidence rate and latent period. To start with the stability of the disease-free and endemic equilibrium of the model with and without delay is dealt with. The existence of Hopf bifurcation is analysed and then obtained by regarding the time delay as the bifurcation parameter. Further, the stochastic model is derived from the deterministic epidemic model through the introduction of random perturbations around the endemic equilibrium point and stochastic stability properties of the model are investigated. The examples and simulations are supplied to throw light on results arrived at.
\end{abstract}

AMS Subject Classification: 92B, 92D, 34D, 70K, 60H

Key Words: SIS Model, Hopf bifurcation, saturation incidence rate, stochastic differential equation, reproductive number

Received: January 14, 2016

Revised: $\quad$ November 4, 2016

Published: February 13, 2017

${ }^{\S}$ Correspondence author
(C) 2017 Academic Publications, Ltd. url: www.acadpubl.eu 


\section{Introduction}

SIR models are predicated on transitions emerging from susceptible $(\mathrm{S})$ to infective (I) to removed (R), with the removal coming through recovery with full immunity (as in measles) or through death from the disease (as in plague, rabies, and many other animal diseases).

Another type of model is an SIS model [1,2] in which infectives go back to the susceptible class on recovery because the disease guarantees no immunity against reinfection. Such models work extremely well with most diseases transmitted by bacterial or helminth agents, and most sexually transmitted diseases (including gonorrhoea, but not such diseases as AIDS, from which there is no recovery). One important and perhaps crucial aspect in which SIS models differ from SIR models is that in the former there is a continuing flow of new susceptible, namely, recovered infectives.

The simplest SIS model, due to Kermack and McKendrick(1932) [13], is

$$
\begin{gathered}
\frac{d S}{d t}=-\beta S I+\gamma I, \\
\frac{d I}{d t}=\beta S I-\gamma I .
\end{gathered}
$$

Mathematical modelling apparently is an important tool to understand and predict the spread of infectious diseases [5]. The rate of incidence very naturally has a crucial role to play in comprehending the processes. It is an epidemiological model that looks at the rate at which a susceptible organism begins to show symptoms of infection too. The representation of incidence rate takes the form made so significant through the classical epidemic Kermack McKendrick model (1927) $\beta S I$, where $S$ and $I$ denote the number of susceptible and infectious, respectively, while $\beta$ is the infection coefficient. The standard incidence is $\beta S I / N$ where $N$ is the total population size and $\beta$ is the daily contact rate. The saturated incidence rate [7-9] assumes the form $\beta S I / 1+\alpha I$, where $\alpha$ is a constant. The effect of saturation factor $(\alpha)$ stems from epidemic control.

This paper sees the adoption of an SIS model with saturated incidence rate described by the following differential system of equations:

$$
\begin{aligned}
\frac{d S}{d t}=r S\left(1-\frac{S}{k}\right) & -\frac{\beta S I}{1+\alpha I}-d S+\gamma I \\
\frac{d I}{d t} & =\frac{\beta S I}{1+\alpha I}-(d+\gamma) I
\end{aligned}
$$

The growth rate of the susceptible organisms depends logistically on density where $r$ is the maximal per capita birth rate; $1 / k$ is the strength of density 
dependence on birth rates, $\beta$ is the transmission rate, $d$ is the natural death rate and $\gamma$ is the recovery rate of the infective individuals. The saturated incidence rate $\beta S I / 1+\alpha I$ was employed by Capasso and Serio [6] in their modelling of cholera, where $\beta S I$ measures the infection force of the disease and $1 / 1+\alpha I$ measures the inhibition effect from the behavioural change of the susceptible individuals when their number increases or as a result of the crowding effect of the infective individuals.

In many infectious diseases, an individual once infected is unable to immediately infect another, and he/she/it undergoes a certain dormant period $(\tau)$ before it can infect others. Mathematically this may be viewed as the delay effect of the infection. Therefore it is interesting to investigate the saturated incidence rates and the latent period.

Hence, system (1) can be represented as:

$$
\begin{array}{r}
\frac{d S}{d t}=r S\left(1-\frac{S}{k}\right)-\frac{\beta S I(t-\tau)}{1+\alpha I(t-\tau)}-d S+\gamma I \\
\frac{d I}{d t}=\frac{\beta S I(t-\tau)}{1+\alpha I(t-\tau)}-(d+\gamma) I
\end{array}
$$

The dynamics of the system (2) are studies in terms of local stability and of the description of the Hopf bifurcation [3, 4, 14].

We consider a stochastic version of the SIS model by generating a slight disturbance in the deterministic system (1) through a white noise [11, 15]. Population systems are often subject to environmental noise; that is, due to environmental fluctuations. Parameters involved in epidemic models are not absolute constants, and they may oscillate or fluctuate around some average values.

Based on these factors, several mathematicians and scientists began to express interest in stochastic epidemic models since these models are capable of providing an additional degree of realism in comparison with their deterministic counterparts and therefore offer a greater degree of flexibility and accuracy.

In epidemic model, randomness in the coefficients may stem from errors in the observed or measured population data, marked variation in the populations and diseases, expected uncertainties such as missing data, or simply from incomplete or incorrect or little awareness of how the system actually works.

The following stochastic differential equation corresponds to model (1)

$$
\begin{array}{r}
\frac{d S}{d t}=r S\left(1-\frac{S}{k}\right)-\frac{\beta S I}{1+\alpha I}-d S+\gamma I+\sigma_{1}\left(S-S^{*}\right) d \xi_{t}^{1} \\
\frac{d I}{d t}=\frac{\beta S I}{1+\alpha I}-(d+\gamma) I+\sigma_{2}\left(I-I^{*}\right) d \xi_{t}^{2}
\end{array}
$$


where $\sigma_{1}, \sigma_{2}$ are real constants and known as the intensity of environmental fluctuations, and $\xi_{t}^{i}=\xi_{i}(t), i=1,2$ are independent of each other in standard Wiener processes.

The paper is structured as follows. In Section 2 we obtain the existence of equilibrium points and their stability with and without delay and we carry out the existence of local Hopf bifurcation in Section 3. Furthermore, we study the dynamical properties of stochastic model (3) by means of Lyapunov functions methods in Section 4. Some numerical simulations aimed at justifying the theoretical analysis are presented in Section 5. Finally, conclusions are presented in Section 6.

\section{Equilibrium States and Their Stability}

The system (2) has always disease free equilibrium $E_{0}$ and unique endemic equilibrium $E_{1}$.

The disease-free equilibrium is given by $E_{0}\left(\frac{k}{r}(r-d), 0\right)$ and it is exists if $r>d$. The endemic equilibrium is the solution of following equations

$$
\begin{gathered}
r S\left(1-\frac{S}{k}\right)-\frac{\beta S I}{1+\alpha I}-d S+\gamma I=0 \\
\frac{\beta S I}{1+\alpha I}-(d+\gamma) I=0
\end{gathered}
$$

Now from equation (5) $S^{*}=(d+\gamma)(1+\alpha I) / \beta$ and substituting $S^{*}$ in (4), we get

$r(d+\gamma) \alpha^{2} I^{2}+\left[2 r(d+\gamma)+k \beta d+\left(k \beta^{2} d / \alpha\right)-k \beta r\right] \alpha I+r(d+\gamma)+k \beta d-k \beta r=0$

we define the basic reproduction number as follows

$$
R_{0}=\frac{k \beta r}{r(d+\gamma)+k \beta d}
$$

From equation (6) we see that if $R_{0} \leq 1$, there is no positive solution as in that case coefficient of $I^{2}, I$ and constant term are all positive, but if $R_{0} \geq 1$, then by Descartes rule there exists a unique positive solution of (6) and consequently there exists unique positive equilibrium $E_{1}\left(S^{*}, I^{*}\right)$ called endemic equilibrium.

Therefore

$$
I^{*}=\frac{-\Delta_{2}+\sqrt{\Delta_{2}^{2}-4 \Delta_{1} \Delta_{3}}}{2 \Delta_{1}}
$$


where

$$
\begin{array}{r}
\Delta_{1}=r(d+\gamma) \alpha^{2}, \quad \Delta_{2}=\left[2 r(d+\gamma)+k \beta d+\left(k \beta^{2} d / \alpha\right)-k \beta r\right] \alpha \\
\Delta_{3}=r(d+\gamma)+k \beta d-k \beta r
\end{array}
$$

obviously $\Delta_{2}^{2}-4 \Delta_{1} \Delta_{3}>0$, when $R_{0}>1$

\subsection{Disease-Free Equilibrium and Its Stability}

The Jacobian matrix of the linearized system of model $(2)$ at $E_{0}\left(S^{*}, 0\right)$ is given by

$$
J=\left[\begin{array}{cc}
r-\frac{2 r S^{*}}{k}-d & -\beta S^{*} e^{-\lambda \tau}+\gamma \\
0 & \beta S^{*} e^{-\lambda \tau}-d-\gamma
\end{array}\right]
$$

with the characteristic equation

$$
\begin{gathered}
\left(\lambda-r+\frac{2 r S^{*}}{k}+d\right)\left(\lambda-\beta S^{*} e^{-\lambda \tau}+d+\gamma\right)=0 . \\
\text { At } E_{0}\left(\frac{k}{r}(r-d), 0\right) \text { above equation reduces to } \\
(\lambda+r-d)\left(\lambda-\frac{\beta k}{r}(r-d) e^{-\lambda \tau}+d+\gamma\right)=0
\end{gathered}
$$

In case of $\tau=0$ (10) has two real and negative roots are if $r>d$ and $R_{0}<1$. In case of $\tau>0$, we assume that the root of (10) $\tau=\omega i$ must satisfy

$$
\omega^{2}=\left(\frac{k \beta d+r(d+\gamma)}{r^{2}}\right)[k \beta(r-d)+r(d+\gamma)]\left[R_{0}-1\right]
$$

Then, when $r>d$ and $R_{0}<1$, then there is no positive real roots $\omega$. Therefore, we arrive the following theorem to indicate the stability of $E_{0}$.

Theorem 1. The disease-free equilibrium $E_{0}\left(\frac{k}{r}(r-d), 0\right)$ of system (2) is asymptotically stable when $r>d$ and $R_{0}<1$ and unstable when $R_{0}>1$.

\subsection{Endemic Equilibrium and Its Stability}

Now the Jacobian matrix $J$ at endemic equilibrium $E_{1}\left(S^{*}, I^{*}\right)$ is given by

$$
J=\left[\begin{array}{cc}
r-\frac{2 r S^{*}}{k}-\frac{\beta I^{*}}{1+\alpha I^{*}}-d & -\frac{\beta S^{*} e^{-\lambda \tau}}{\left(1+\alpha I^{*}\right)^{2}}+\gamma \\
\frac{\beta I^{*}}{1+\alpha I^{*}} & \frac{\beta S^{*} e^{-\lambda \tau}}{\left(1+\alpha I^{*}\right)^{2}}-d-\gamma
\end{array}\right]
$$


The characterstic equation of (11) is given by

$$
\lambda^{2}+P_{1} \lambda+P_{2}+e^{-\lambda \tau}\left(Q_{1} \lambda+Q_{2}\right)=0
$$

where $\quad P_{1}=\frac{2 r S^{*}}{k}+\frac{\beta I^{*}}{1+\alpha I^{*}}+2 d+\gamma-r$

$$
\begin{aligned}
& P_{2}=r(d+\gamma)+\frac{2 r S^{*}}{k}(d+\gamma)+d(d+\gamma)+\frac{\beta d I^{*}}{1+\alpha I^{*}} \\
& Q_{1}=-\frac{\beta S^{*}}{\left(1+\alpha I^{*}\right)^{2}} \quad \& \quad Q_{2}=\frac{r \beta S^{*}}{\left(1+\alpha I^{*}\right)^{2}}-\frac{2 r \beta S^{* 2}}{k\left(1+\alpha I^{*}\right)^{2}}-\frac{d \beta S^{*}}{\left(1+\alpha I^{*}\right)^{2}}
\end{aligned}
$$

we need to find the necessary and sufficient condition for every root of the characteristic equation (12) having negative real part.

Case 1: For $\tau=0$, (12) becomes

$$
\lambda^{2}+\left(P_{1}+Q_{1}\right) \lambda+\left(P_{2}+Q_{2}\right)=0
$$

where $R_{0}>1$, we have $P_{1}+Q_{1}>0 \quad \& \quad P_{2}+Q_{2}>0$.

By Routh-Hurwitz criteria, all roots of (13) are real and negative or complex conjugate with negative real part.

Hence the system (2) without delay is locally asymptotically stable when $R_{0}>1$.

Case 2: If $\tau>0$, suppose that there is a positive $\tau_{0}$ such that equation (12) has pair of purely imaginary roots $\pm i \omega, \omega>0$. Then $\omega$ satisfies

$$
-\omega^{2}+P_{1} \omega i+P_{2}+\left(Q_{1} \omega i+Q_{2}\right)[\cos \omega t-i \sin \omega t]=0
$$

separating the real and imaginary parts, we have

$$
\begin{aligned}
& \omega^{2}-P_{2}=Q_{1} \omega \sin \omega t+Q_{2} \cos \omega t \\
& -P_{1} \omega=-Q_{2} \sin \omega t+Q_{1} \omega \cos \omega t
\end{aligned}
$$

which is equivalent to

$$
\omega^{4}+\left(P_{1}^{2}-2 P_{2}-Q_{1}^{2}\right) \omega^{2}+\left(P_{2}^{2}-Q_{2}^{2}\right)=0
$$

If $P_{1}^{2}-2 P_{2}-Q_{1}^{2}>0, \quad P_{2}^{2}-Q_{2}^{2}>0$, equation (16) has no real root. Thus the real parts of all Eigen values of (12) are negative for all $\tau \geq 0$. Hence endemic equilibrium $E_{1}$ is asymptotically stable for all $\tau$ if the following conditions hold:

$$
\begin{aligned}
& \text { i). } R_{0}>1 \\
& \text { ii). }\left(P_{1}+Q_{1}\right)>0,\left(P_{2}+Q_{2}\right)>0 \\
& \text { iii). } P_{1}^{2}-2 P_{2}-Q_{1}^{2}>0, P_{2}^{2}-Q_{2}^{2}>0
\end{aligned}
$$


If $P_{2}^{2}-Q_{2}^{2}$ is negative, there is a unique positive $\omega_{0}$ satisfying (16) and then there is a positiive $\tau_{0}$ such that equation (12) has pair of purely imaginary roots $\pm i \omega_{0}$ as $\tau=\tau_{0}$, and all eigen values with negative real parts as $0<\tau<\tau_{0}$.

From (15) $\tau_{k}$ corresponding to $\omega_{0}$ can be obtained

$$
\tau_{k}=\frac{1}{\omega_{0}} \arccos \left[\frac{\left(Q_{2}-P_{1} Q_{1}\right) \omega^{2}-P_{2} Q_{2}}{Q_{1}^{2} \omega^{2}+Q_{2}^{2}}\right]+\frac{2 n \pi}{\omega_{0}}, n=0,1,2 \ldots .
$$

\section{Hopf Bifurcation}

Based on the above results, we have the following

Theorem 2. Assume that $R_{0}>1$ then there is a positive $\tau_{0}$ such that the following results hold.

(i) If $0<\tau<\tau_{0}$, equation (2) has an endemic equilibrium $E_{1}$ which is locally asymptotically stable.

(ii) Equation (2) can undergo a Hopf bifurcation if $\tau>\tau_{0}$, and a periodic orbit exists in the small neighbourhood of the endemic equilibrium.

Proof. To obtain the Hopf bifurcation, we need to check the transversal condition for the complex eigen values of the $E_{1}$ at $\tau=\tau_{0}$. Then, from equation (12), we have

$$
\begin{aligned}
& \frac{d \lambda}{d t}\left[2 \lambda+P_{1}+Q_{1} e^{-\lambda \tau}-\left(Q_{1} \lambda+Q_{2}\right) \tau e^{-\lambda \tau}\right]=\lambda\left(Q_{1} \lambda+Q_{2}\right) e^{-\lambda \tau} \\
& \left(\frac{d \lambda}{d t}\right)^{-1}=\frac{2 \lambda+P_{1}+Q_{1} e^{-\lambda \tau}-\left(Q_{1} \lambda+Q_{2}\right) \tau e^{-\lambda \tau}}{\lambda\left(Q_{1} \lambda+Q_{2}\right) e^{-\lambda \tau}} \\
& \left(\frac{d \lambda}{d t}\right)^{-1}=\frac{2 \lambda+P_{1}}{-\lambda\left(\lambda^{2}+P_{1} \lambda+P_{2}\right)}+\frac{Q_{1}}{\lambda\left(Q_{1} \lambda+Q_{2}\right)}-\frac{\tau}{\lambda} \\
& \left.\frac{d R e(\lambda)}{d t}\right|_{\lambda=i \omega_{0}}=\left.\operatorname{Re}\left(\frac{d \lambda}{d t}\right)^{-1}\right|_{\lambda=i \omega_{0}} \\
& =\operatorname{Re}\left[\frac{2 i \omega_{0}+P_{1}}{-i \omega_{0}\left(-\omega_{0}^{2}+P_{1} i \omega_{0}+P_{2}\right)}+\frac{Q_{1}}{i \omega_{0}\left(Q_{1} i \omega_{0}+Q_{2}\right)}-\frac{\tau}{i \omega_{0}}\right] \\
& =\operatorname{Re}\left[\frac{1}{\omega_{0}}\left(\frac{2 i \omega_{0}+P_{1}}{P_{1} \omega_{0}+\left(\omega_{0}^{2}-P_{2}\right) i}+\frac{Q_{1}}{\left(-Q_{1} \omega_{0}+Q_{2} i\right)}+\tau i\right)\right]
\end{aligned}
$$




$$
\begin{aligned}
& =\frac{1}{\omega_{0}}\left(\frac{2 \omega_{0}\left(\omega_{0}^{2}-P_{2}\right)+P_{1}^{2} \omega_{0}}{P_{1}^{2} \omega_{0}+\left(\omega_{0}^{2}-P_{2}\right)^{2}}-\frac{Q_{1}^{2}}{\left(Q_{1}^{2} \omega_{0}^{2}+Q_{2}^{2}\right)}\right) \\
& =\frac{2 \omega_{0}^{2}+\left(P_{1}^{2}-2 P_{2}-Q_{1}^{2}\right)}{\left(Q_{1}^{2} \omega_{0}^{2}+Q_{2}^{2}\right)}
\end{aligned}
$$

Under the condition $P_{1}^{2}-2 P_{2}-Q_{1}^{2}>0$, we have $\left.\frac{d \operatorname{Re}(\lambda)}{d t}\right|_{\lambda=i \omega_{0}}>0$.

Therefore, the transversality condition holds and Hopf bifurcation occurs at $\omega=\omega_{0}, \quad \tau=\tau_{0}$.

\section{Stochastic Stability of The Model at Endemic Equilibrium}

To study the environmental fluctuations on model (1), we assume that the stochastic perturbations are of white noise type and that they are proportional to the distances of $S$ and $I$ respectively. The system (3) has the same equilibria as the system (1).

The stochastic differential system (3) can be centered at its positive equilibrium $E_{1}$ by the change of variables

$$
u_{1}=S-S^{*}, \quad u_{2}=I-I^{*}
$$

The linearized Stochastic Differential Equations around $E_{1}$ take the form

$$
d u(t)=f(u(t)) d t+g(u(t)) d \xi(t)
$$

where $u(t)=\left(u_{1}(t), u_{2}(t)\right)^{T}$

$$
\begin{gathered}
f(u(t))=\left[\begin{array}{cc}
r-\frac{2 r S^{*}}{k}-\frac{\beta I^{*}}{1+\alpha I^{*}}-d & \frac{-\beta S^{*}}{\left(1+\alpha I^{*}\right)^{2}}+\gamma \\
\frac{\beta I^{*}}{1+\alpha I^{*}} & \frac{\beta S^{*}}{\left(1+\alpha I^{*}\right)^{2}}-d-\gamma
\end{array}\right] \\
g(u)=\left[\begin{array}{cc}
\sigma_{1} u_{1} & 0 \\
0 & \sigma_{2} u_{2}
\end{array}\right]
\end{gathered}
$$

Let $C^{1,2}\left([0,+\infty) \times \Re^{2}, \Re^{+}\right)$be the family of nonnegative functions. $W(t, u)$ defined on $\left([0,+\infty) \times \Re^{2}\right.$ is a continuously differentiable function with respect to $t$ and twice with respect to $u$.

We define the differential operator $L$ for a function $W(t, u)$ by

$$
L W(t, u)=\frac{\partial W(t, u)}{\partial t}+f^{T}(u) \frac{\partial W(t, u)}{\partial u}+\frac{1}{2} \operatorname{Tr}\left[g^{T}(u) \frac{\partial^{2} W(t, u)}{\partial u^{2}} g(u)\right]
$$




$$
\begin{gathered}
\frac{\partial W}{\partial u}=\operatorname{col}\left(\frac{\partial W}{\partial u_{1}}, \frac{\partial W}{\partial u_{2}}, \frac{\partial W}{\partial u_{3}}\right) \\
\frac{\partial^{2} W(t, u)}{\partial u^{2}}=\left(\frac{\partial^{2} W}{\partial u_{j} \partial u_{i}}\right) \quad i, j=1,2 \text { and }{ }^{\prime} T^{\prime} \text { means transposition. }
\end{gathered}
$$

with reference to the book by Afanas'ev et al. [10], the following theorem holds.

Theorem 3. Suppose there exists a function $W(t, u) \in C^{1,2}([0,+\infty) \times$ $\left.\Re^{2}, \Re^{+}\right)$satisfying the following inequalities

$$
\begin{array}{r}
K_{1}|u|^{p} \leq W(t, u) \leq K_{2}|u|^{p} \\
L W(t, u) \leq-K_{3}|u|^{p},
\end{array}
$$

where $K_{1}, K_{2}, K_{3}$ and $p$ are positive constants.

Then the trivial solution of (20) is exponentially $p$ - stable for $t \geq 0$. Moreover, if in (23), $p=2$ the trivial solution of (20) is globally asymptotically stable in probability.

Theorem 4. Suppose that

$$
\sigma_{1}^{2} \leq 2\left(\frac{2 r S^{*}}{k}+\frac{\beta I^{*}}{1+\alpha I^{*}}+d-r\right), \sigma_{2}^{2} \leq 2\left((d+\gamma)-\frac{\beta S^{*}}{\left(1+\alpha I^{*}\right)^{2}}\right) \text { hold } .
$$

Then, the trivial solution of (20) is asymptotically mean square stable.

Proof. Let us consider the Lyapunov function

$$
W(u)=\frac{1}{2}\left[w_{1} u_{1}^{2}+w_{2} u_{2}^{2}\right]
$$

where $w_{1}, w_{2}$ are non-negative constants to be chosen in the following. It is easy to check that inequalities (23) hold true with $p=2$.

$$
\begin{gathered}
L W(u)=w_{1}\left[\left(r-\frac{2 r S^{*}}{k}-\frac{\beta I^{*}}{1+\alpha I^{*}}-d\right) u_{1}+\left(\gamma-\frac{\beta S^{*}}{\left(1+\alpha I^{*}\right)^{2}}\right) u_{2}\right] u_{1} \\
+w_{2}\left[\frac{\beta I^{*}}{1+\alpha I^{*}} u_{1}+\left(\frac{\beta S^{*}}{\left(1+\alpha I^{*}\right)^{2}}-(d+\gamma)\right) u_{2}\right] u_{2}+\frac{1}{2} \operatorname{Tr}\left[g^{T}(u) \frac{\partial^{2} W(t, u)}{\partial u^{2}} g(u)\right] \\
\text { with } \frac{1}{2} \operatorname{Tr}\left[g^{T}(u)+\frac{\partial^{2} W(t, u)}{\partial u^{2}} g(u)\right]=\frac{1}{2}\left[w_{1} \sigma_{1}^{2} u_{1}^{2}+w_{2} \sigma_{2}^{2} u_{2}^{2}\right]
\end{gathered}
$$

If in $(25)$ we choose $\left(\frac{\beta S^{*}}{\left(1+\alpha I^{*}\right)^{2}}-\gamma\right) w_{1}=\frac{\beta I^{*}}{\left(1+\alpha I^{*}\right)} w_{2}, \quad$ then 


$$
\begin{array}{r}
L W(u)=-\left(\frac{2 r S^{*}}{k}+\frac{\beta I^{*}}{1+\alpha I^{*}}+d-r-\frac{1}{2} \sigma_{1}^{2}\right) w_{1} u_{1}^{2}-\left((d+\gamma)-\frac{\beta S^{*}}{\left(1+\alpha I^{*}\right)^{2}}\right. \\
\left.-\frac{1}{2} \sigma_{2}^{2}\right) w_{2} u_{2}^{2}
\end{array}
$$

According to Theorem (3), we conclude that the trivial solution of system (20) is globally asymptotically stable.

\section{Numerical Simulations}

In this section, we substantiate as well as augment our analytical results through numerical simulations considering the following examples.

Example 1. We take the following parameters. $r=1.9 ; k=79.6 ; d=$ $0.964 ; \beta=0.47 ; \alpha=0.102 ; \gamma=0.068$.

System (2) has the unique positive equilibrium $E_{1}(2.7312,2.3906)$ and $R_{0}=$ $1.8693>1$. It follows from result (18), that the critical positive time delay $\tau_{0}=0.5499$ and we know that when $0 \leq \tau<\tau_{0}, E_{1}$ is asymptotically stable. From the Theorem (2) when $\tau$ passes through the critical value $\tau_{0}=0.5499$, the positive equilibrium loses its stability and a family of periodic solutions bifurcate from $E_{1}$. (Fig.1-3)

Example 2. We numerically simulate the dynamics of the deterministic and the stochastic model system around the positive interior steady state for the above set of parameter values with $\sigma_{1}=0.02, \sigma_{2}=0.01$. It is easy to see that, all the conditions of Theorem (4) are satisfied. Fig (4-5) represents deterministic and stochastic trajectories of the system (3).

\section{Conclusion}

From the above results and numerical examples the following conclusions may be inferred: The disease-free equilibrium exists only if $r>d$ and asymptotically stable when $R_{0}<1$. When $R_{0}>1$, endemic equilibrium is achieved and the population is asymptotically stable. We showed that the local stability of the endemic equilibrium point $E_{1}$ depends on the time delay $\tau$. The system changes behaviour from stable to unstable nature around $E_{1}$ when $\tau$ crosses the critical value $\tau_{0}$ via a Hopf bifurcation from $E_{1}$. The model in question here supplies evidence to the effect that the stochastic model is globally asymptotically stable in probability when the intensities of white noise are less than certain threshold 

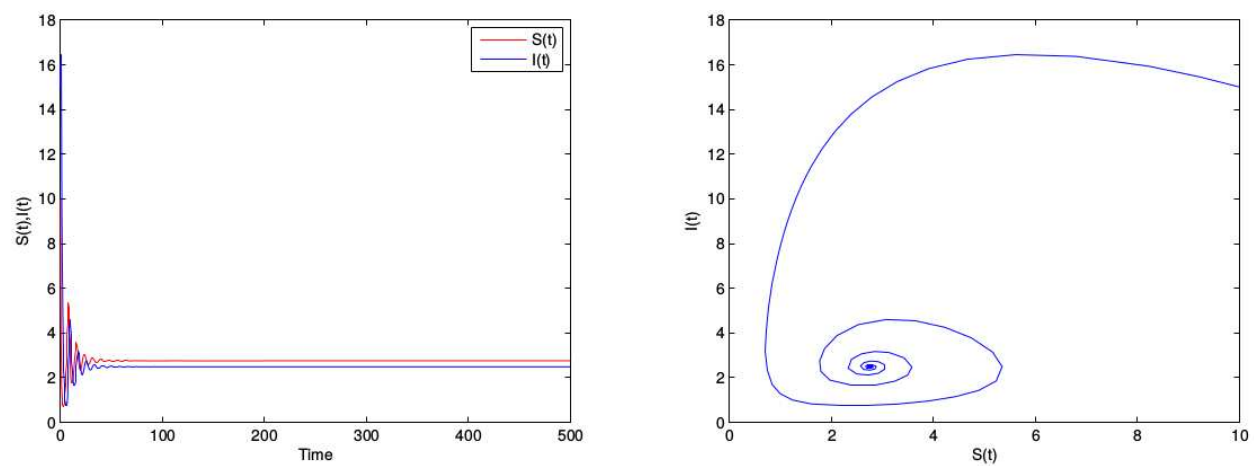

Figure 1: The trajectories and graphs of system (2) with $\tau=0.1<\tau_{0}=$ 0.5499
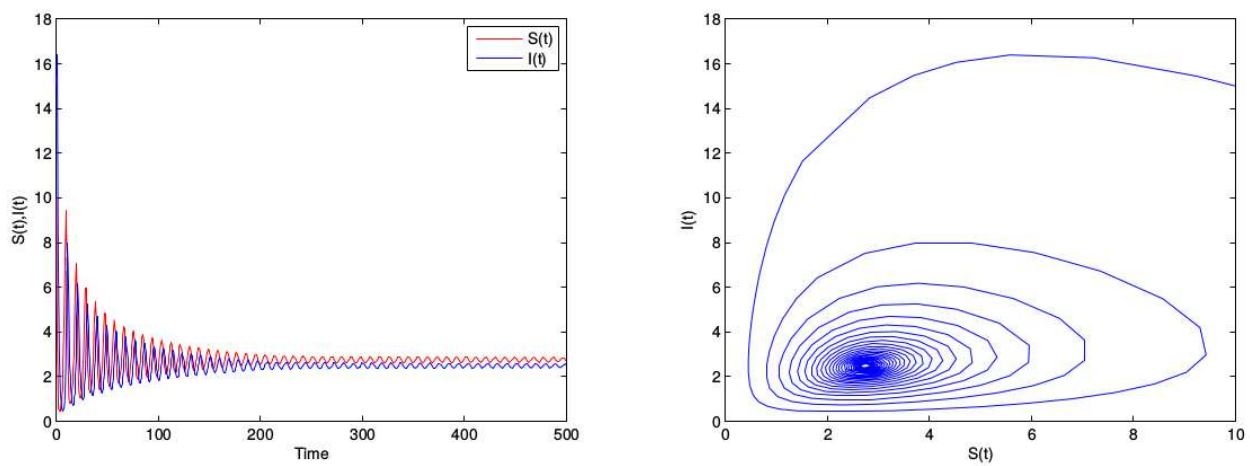

Figure 2: The trajectories and phase graphs of system (2) with $\tau=$ $\tau_{0}=0.5499$

parameters. Finally, numerical simulations have been factored in to verify the analytical results and prove beyond doubt the reliability and validity of the mathematical model assumed for explaining the phenomenon.

\section{References}

[1] H.W. Hethcote and P. Van den Driessche, An SIS epidemic model with variable population size and a dela, Journal of Mathematical Biology, 34, No. 2 (1995), 177-194, doi: $10.1007 / \mathrm{bf00178772.}$

[2] H.W. Hethcote, P. Van den Driessche, Two SIS epidemiologic models with delays, Journal of Mathematical Biology, 40 (2000), 3-26, doi: 10.1007/bf00160539. 

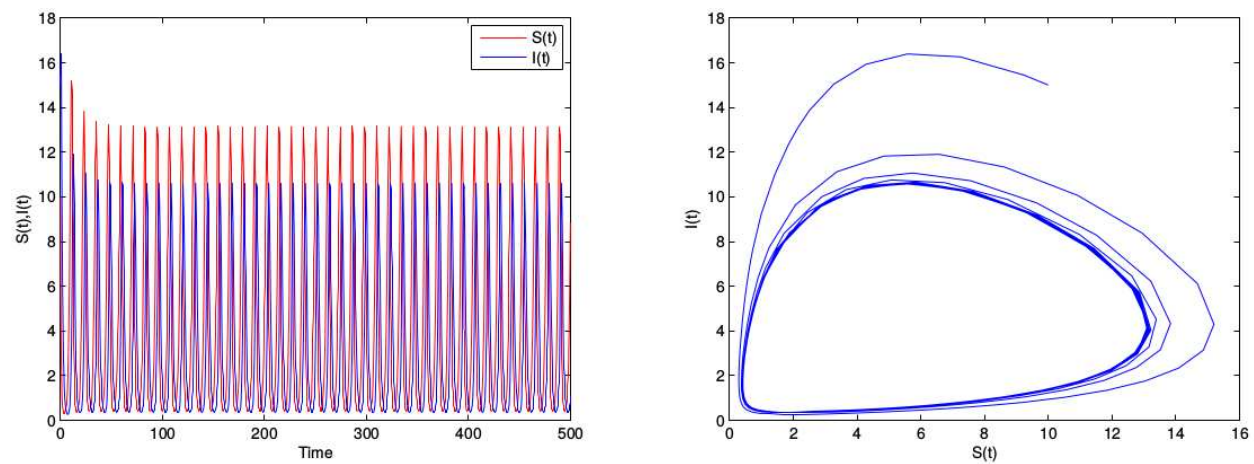

Figure 3: The trajectories and phase graphs of system (2) with $\tau=$ $1>\tau_{0}=0.5499$
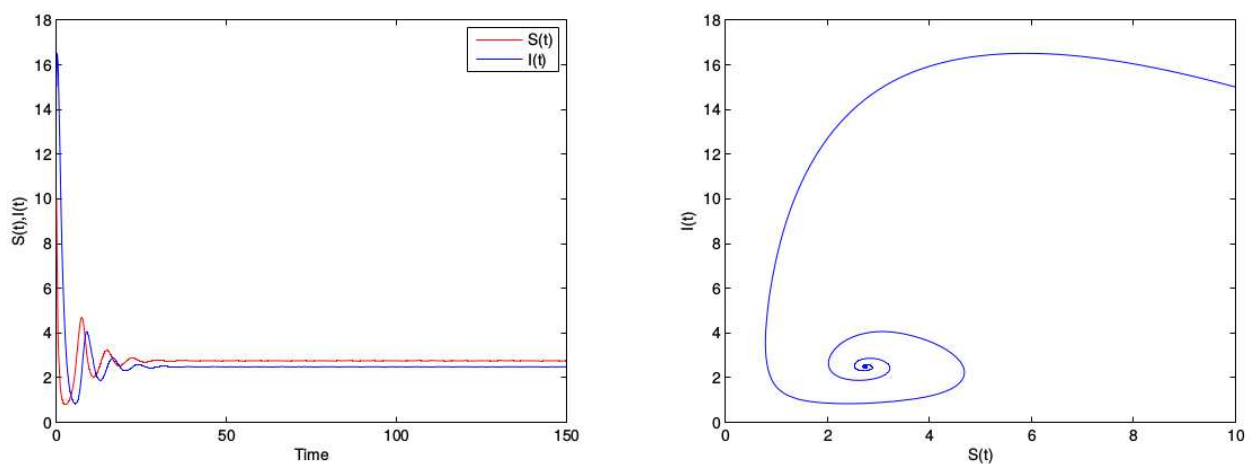

Figure 4: represents deterministic trajectories and phase graphs of model (3)

[3] K. Gopala Swamy, Stability and Oscillations in Delay Differential Equations of Population Dynamics, Springer, Kluwer Academic Publishers, Netherlands, 1992, doi: 10.1007/978-94-015-7920-9.

[4] Yang Kuang, Delay Differential Equations with Applications in Population Dynamics, Academic Press, New York, 1993, doi: 10.1016/s0076-5392(08)62861-1.

[5] R.M. Anderson and R.M. May, Population biology of infectous diseases, Nature, 280, 5721 (1979), 361-367, doi: 10.1038/280361a0.

[6] V. Capasso, G. Serio, A generalization of the Kermack-Mckendrick deterministic epidemic model, Math. Biosci, 42 (1978), 65-81, doi: 10.1016/0025-5564(78)90006-8.

[7] S. Ruan and W. Wang, Dynamical behavior of an epidemic model with a nonlinear incidence rate, J. Differential Equations, 188 (2003), 135-163, doi: 10.1016/s00220396(02)00089-x. 

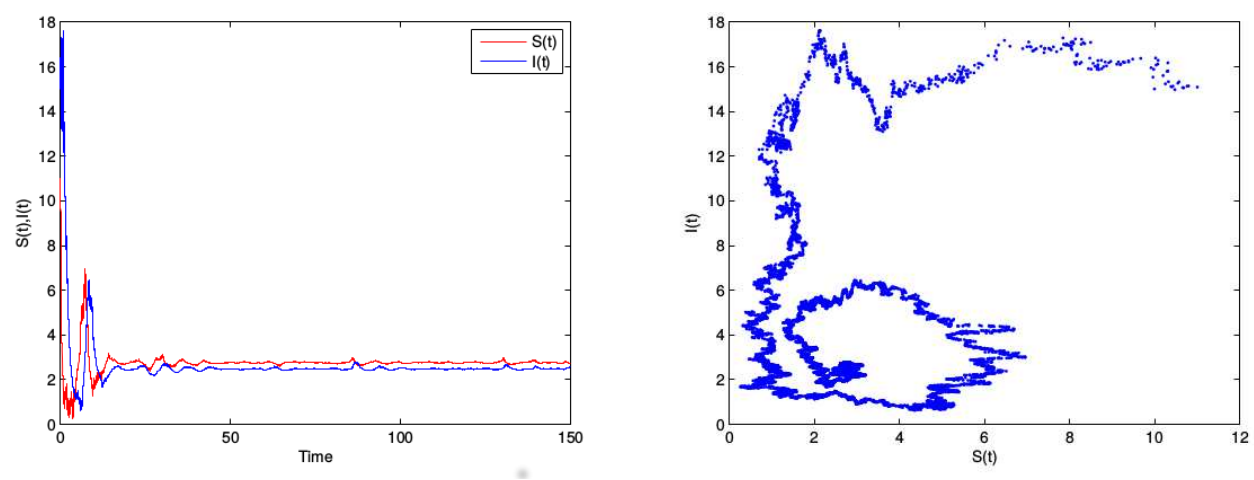

Figure 5: represents stochastic trajectories and phase graphs of model (3)

[8] G. Li and Wang, Bifurcation analysis of an epidemic model with nonlinear incidence, Appl. Math. Comput., 214 (2009), 411-423, doi: 10.1016/j.amc.2009.04.012.

[9] G. Ranjith Kumar, K. Lakshmi Naryan and B. Ravindra Reddy, Stability analysis of epidemic model with nonlinear incidence rates and immigration, In: Proceedings of $\mathrm{Na}$ tional Conference on Pure and Applied Mathematics, ISBN 978-93-83459-76-9, Bonfring, 69-73.

[10] V.N. Afanasev, V.B. Kolmanowski, and V.R. Nosov, Mathematical Theory of Control System Design, Kluwer Academic, Dordrecht, Netherlands, 1996, doi: 10.1007/978-94017-2203-2.

[11] P. Das, D. Mukherjee, and A. K. Sarkar, Study of an S-I epidemic model with nonlinear incidence rate: Discrete and stochastic version, Applied Mathematics and Computation, 218, No. 6 (2011), 2509-2515, doi: 10.1016/j.amc.2011.07.065.

[12] D. Xiao, S. Ruan, Global analysis of an epidemic model with non-monotone incidence rate, Math. Bioscience, 208 (2007), 419-429, doi: 10.1016/j.mbs.2006.09.025.

[13] W.O. Kermack, A.G. McKendrick, Contribution to mathematical theory of epidemics, P. Roy. Soc. Lond., Mat., 115 (1927), 700-721, doi: 10.1098/rspa.1927.0118.

[14] L.S. Chen, J. Chen, Nonlinear Biological Dynamics System, Scientific Press, China, 1993.

[15] L.J.S. Allen, An introduction to stochastic epidemic models, In: Mathematical Epidemiology (Ed-s: F. Brauer, P. Van den Driessche, and J. Wu) (1945), 81-130, Springer, Berlin, Germany, (2008), doi: 10.1007/978-3-540-78911-6_3. 
